\title{
Preoperative neutrophil-to-lymphocyte ratio predicts the surgical outcome of Xp11.2 translocation/TFE3 renal cell carcinoma patients
}

Sezim Agizamhan ${ }^{1 \dagger}$, Feng Qu ${ }^{1 \dagger}$, Ning Liu', Jing Sun², Wei Xu³, Lihua Zhang ${ }^{4}$, Hongqian Guo ${ }^{1}$ and Weidong Gan ${ }^{1 *}$

\begin{abstract}
Background: The preoperative neutrophil-to-lymphocyte ratio (NLR), C-reactive protein/albumin ratio (CRP/Alb ratio) and platelet-to-lymphocyte ratio (PLR) have been demonstrated to predict the clinical outcome of various human cancer, including renal cell carcinoma(RCC). The aim of our study was to explore the prognostic values of these ratios in patients with Xp11.2 translocation/TFE3 gene fusions renal cell carcinoma (Xp11.2 tRCC).

Methods: A retrospective multicentre study was performed in 82 Xp11.2 tRCC patients who underwent radical or partial nephrectomy. The optimal cutoff values of the NLR, CRP/Alb ratio and PLR were determined by the receiver operating characteristic (ROC) analysis. The impact of the NLR, CRP/Alb ratio and PLR, as well as other clinicopathological characteristics, on disease-free survival (DFS) and overall survival (OS) were evaluated using the univariate and multivariate Cox regression analyses.

Results: The optimal cutoff values of the NLR, CRP/Alb ratio and PLR were set at 2.45, 140 and 0.08, respectively, according to the ROC analysis. Univariate analyses showed that the NLR, CRP/Alb ratio and PLR all were associated with DFS of Xp11.2 tRCC patients ( $P<0.001, P=0.005$ and $P=0.001$, respectively) and OS of Xp11.2 tRCC patients $(P=0.016, P=0.003$ and $P=0.014$, respectively). Multivariate analysis indicated that the NLR was independently associated with DFS of Xp11.2 tRCC patients (hazard ratio [HR]: 4.25; 95\% confidence interval [95\% Cl]: 1.19-15.18; $P=0.026$ ) along with age $(P=0.004)$, the $p T$ status $(P<0.001)$ and the $p N$ status $(P<0.019)$, and the NLR (HR: 26.26; 95\% Cl: 1.44-480.3; $P=0.028)$ also was independently associated with OS in patients with Xp11.2 tRCC, along with age $(P=0.016)$ and a tumour thrombus $(P=0.007)$.

Conclusion: Overall, relatively high NLRs, CRP/Alb ratios and PLRs were associated with a poor prognosis of Xp11.2 tRCC patients; among of them, only the NLR independently predicted the progression of Xp11.2 tRCC, and the NLR may help to identify patients with high metastasis or relapse risk.
\end{abstract}

Keywords: Neutrophil-to-lymphocyte ratio, C-reactive protein/albumin ratio, Platelet-to-lymphocyte ratio, Renal cell carcinoma, Xp11.2 translocation

\footnotetext{
*Correspondence: gwd@nju.edu.cn

${ }^{\dagger}$ Sezim Agizamhan and Feng Qu contributed equally to this work.

'Department of Urology, Nanjing Drum Tower Hospital, The Affiliated

Hospital of Nanjing University Medical School, No. 321 Zhongshan Road,

Nanjing 210008, Jiangsu Province, China

Full list of author information is available at the end of the article
}

(c) The Author(s). 2018 Open Access This article is distributed under the terms of the Creative Commons Attribution 4.0 International License (http://creativecommons.org/licenses/by/4.0/), which permits unrestricted use, distribution, and reproduction in any medium, provided you give appropriate credit to the original author(s) and the source, provide a link to the Creative Commons license, and indicate if changes were made. The Creative Commons Public Domain Dedication waiver (http://creativecommons.org/publicdomain/zero/1.0/) applies to the data made available in this article, unless otherwise stated. 


\section{Background}

Xp11.2 translocation/TFE3 gene fusions renal cell carcinoma (Xp11.2 tRCC) was first listed as a new type of renal cell carcinoma (RCC) in the 2004 by the World Health Organization (WHO). Since then, it has received wide attention [1-5]. Xp11.2 tRCC is characterized by various translocations of the transcription factor E3(TFE3)

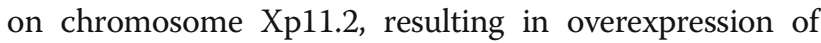
the TFE3 protein [6]. Xp11.2 tRCC is a kind of relatively rare tumour that predominantly occurs in children and young adults [7]. Regardless of its low incidence, Xp11.2 $\mathrm{RCC}$ is more aggressive than conventional RCC because most adult present at advanced stages and invasive clinical courses [3, 4]. Therefore, it is crucial to identify new preoperative prognostic factors to provide additional prognostic information for Xp11.2 tRCC patients. In addition, in regard to the risk of disease recurrence, is important to obtain prognostic information in the preoperative phase for the postoperative surveillance and possible adjuvant therapy.

It is recognized that the inflammatory processes in the tumour microenvironment play a significant role in promoting the proliferation, invasion and metastasis of the malignant cells $[8,9]$. Inflammatory markers, such as the neutrophil count(NC), lymphocyte count(LC), platelet count (PLT), neutrophil-to-lymphocyte ratio (NLR), C-reactive protein (CRP), albumin (Alb), C-reactive protein/ albumin ratio (CRP/Alb ratio) and platelet-to- lymphocyte ratio (PLR), have been shown to predict the clinical outcome of various human cancers [10-13]. For renal cell carcinoma, several publications demonstrated that high NLR, CRP/Alb ratios and PLRs were associated with a poor prognosis in RCC, respectively [14-17].

To our knowledge, the prognostic value of inflammatory markers has never been investigated in the Xp11.2 tRCC patients. Additionally, compared with conventional RCC, Xp11.2 tRCC involves different genetic characteristics and biological pathways and is associated with a more worse prognosis $[3,5,18]$. In addition, inflammatory markers are more easily accessible than other prognostic factors before surgery. Therefore, there is a need to identify new preoperative prognostic markers to predict the clinical outcomes of surgical Xp11.2 tRCC patients. The aims of the present study were to examine the prognostic values of the NLR, CRP/Alb ratio and PLR in patients with Xp11.2 tRCC.

\section{Methods \\ Patients}

Institutional review board approval was obtained at Nanjing Drum Tower Hospital, Jiangsu Province Hospital, Jiangsu Cancer Hospital and Zhongda Hospital Southeast University for this multicentre retrospective study. All the patients have provided informed written consents to have their medical record data used in research. Between January 2007 and July 2017, 89 consecutive patients from the 4

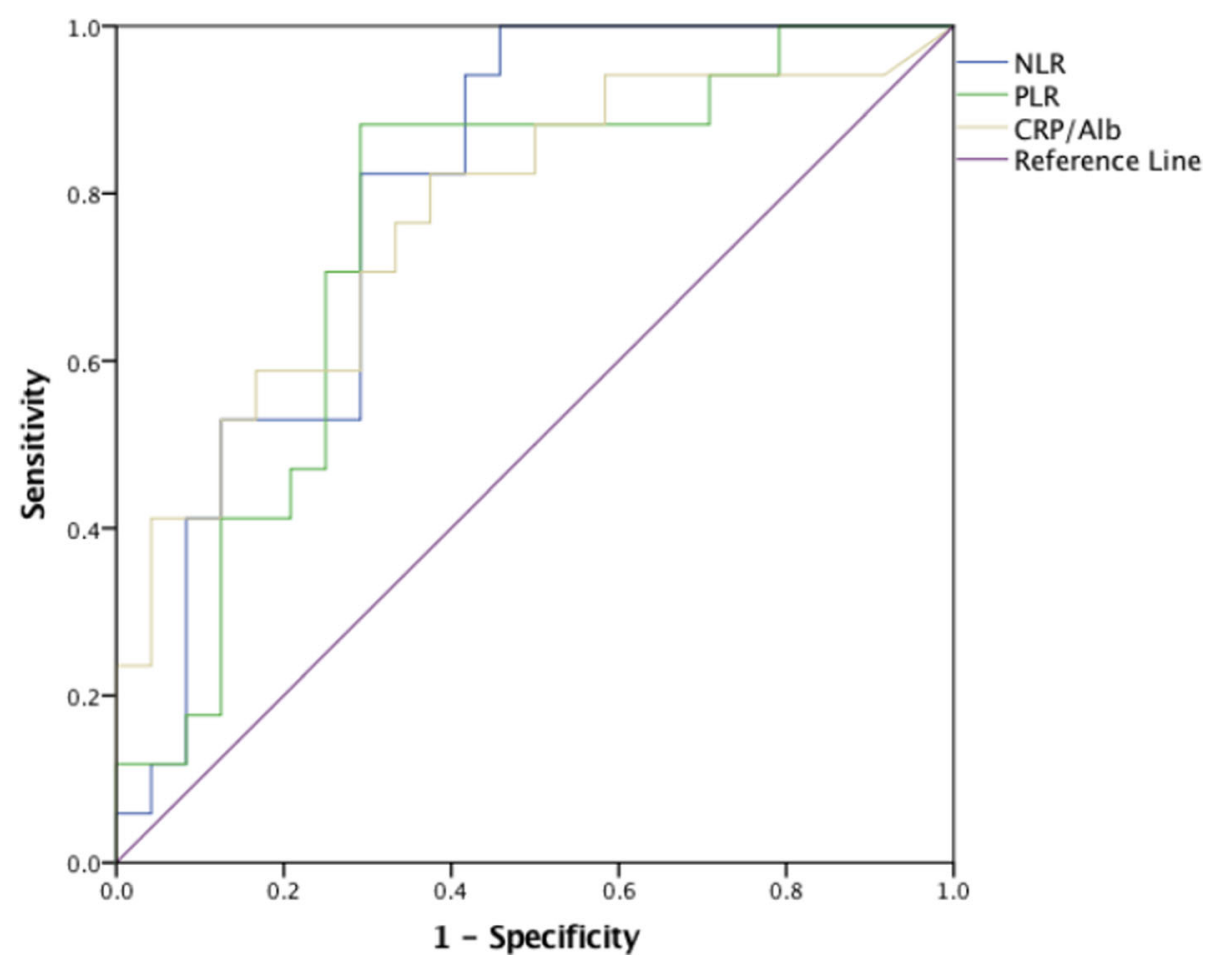

Fig. 1 The predictive abilities of the preoperative NLR, CRP/Alb ratio and PLR were compared using ROC curves 
institutions described above who were diagnosed with Xp11.2 tRCC after radical or partial nephrectomy for a renal mass were reviewed for the present study. All clinicopathological data were retrieved from medical records from the department of urology as well as from pathology reports from the Institute of Pathology at each institution. The inclusion criteria included the following: 1) patients who were histologically and immunohistochemically (using the TFE3 protein nuclear stain) diagnosed with Xp11.2 tRCC; 2) the data on complete blood laboratory tests included the serum neutrophil count(NC), lymphocyte count(LC), platelet count (PLT), C-reactive protein (CRP) level and albumin (Alb) level within one week before performing radical or partial nephrectomy; and (3) patients without blood laboratory tests before surgery, patients with active inflammatory disease and patients with other tumours were excluded from the study. Finally, a total of 82 patients were enrolled in this study.

\section{Clinical and pathological evaluation}

The baseline clinical characteristics and pathologic information, including data on the age at the time of surgery, gender, tumour location, tumour size, symptoms at presentation, surgical treatment, pathological features, immunohistochemistry results, NC, LC, PLT, CRP level, Alb level, lactate dehydrogenase (LDH) level, urine protein, tumour stage, and nuclear grade, were all collected. Tumour stage was determined according to the seventh edition of the TNM-UICC/AJCC classification system, and the nuclear grade was defined based on the Fuhrman Grading System. The NLR was defined as the ratio of the NC to LC. The PLR was defined as the ratio of the PLT to LC. The $\mathrm{CRP} / \mathrm{Alb}$ ratio was defined as the ratio of the serum CRP level to the serum albumin (Alb) level. Elevated LDH was defined as serum $\mathrm{LDH}>245 \mathrm{U} / \mathrm{L}$. The association between inflammatory parameters (LN, NC, PLT, CRP, NLR, PLR, $\mathrm{CRP} / \mathrm{Alb}$ ) and DFS was explored, and the ROC curves of the NLR, CRP/Alb ratio and PLR are shown in Fig. 1.

\section{Patients follow-up}

All patients enrolled in this study were followed-up every 3 months during the first 2 years, every 6 months for 3-5 years and every 12 months after 5 years until July 2017 or until death. A physical examination, laboratory tests, and dynamic computed tomography were performed at every visit. Overall survival (OS) was defined as the time interval between the date of surgery and the date of death or the last follow-up. Disease-free survival (DFS) was defined as the time interval between the date of surgery and date of disease recurrence or metastasis or the last follow-up in localized Xp11.2 tRCC patients who underwent radical or partial nephrectomy.

\section{Statistical analyses}

Statistical analyses were performed using SPSS version 24.0 software (SPSS, Chicago, IL, USA). The descriptive data (i.e., tumour size) were presented as the means \pm standard deviation or medians, and Student's t-test was used for these variables. A comparison between groups was performed using the Chi-squared test. Receiver operating characteristic (ROC) analysis was used for the selection of effective inflammatory parameters and corresponding optimal cut-off values, and the area under the curve (AUC), sensitivity, specificity and $P$ value

Table 1 Baseline characteristics of 82 Xp11.2 tRCC patients

\begin{tabular}{|c|c|}
\hline Characteristic & Case $(n=82)$ \\
\hline \multicolumn{2}{|l|}{ Age in years } \\
\hline$>45$ & 21 \\
\hline$\leq 45$ & 61 \\
\hline \multicolumn{2}{|l|}{ Sex } \\
\hline Male & 33 \\
\hline Female & 49 \\
\hline Tumour size, cm & $5.94 \pm 2.65$ \\
\hline \multicolumn{2}{|l|}{ Location } \\
\hline Left & 31 \\
\hline Right & 51 \\
\hline \multicolumn{2}{|l|}{ Symptoms } \\
\hline Asymptomatic & 48 \\
\hline Symptomatic & 34 \\
\hline \multicolumn{2}{|l|}{ Fuhrman grade } \\
\hline 1 to 2 & 32 \\
\hline 3 to 4 & 50 \\
\hline \multicolumn{2}{|l|}{ TNM stage } \\
\hline | to || & 49 \\
\hline III to IV & 33 \\
\hline \multicolumn{2}{|l|}{ pT status } \\
\hline T1 to $\mathrm{T} 2$ & 64 \\
\hline T3 to T4 & 18 \\
\hline \multicolumn{2}{|l|}{ pN status } \\
\hline No & 59 \\
\hline $\mathrm{N} 1$ & 23 \\
\hline \multicolumn{2}{|l|}{ pM status } \\
\hline MO & 78 \\
\hline M1 & 4 \\
\hline \multicolumn{2}{|l|}{ Surgical treatment } \\
\hline Radical & 55 \\
\hline Partial & 27 \\
\hline \multicolumn{2}{|l|}{ Tumour thrombus } \\
\hline Negative & 72 \\
\hline Positive & 10 \\
\hline
\end{tabular}


Table 2 Clinicopathological features of Xp11.2 tRCC patients stratified by the cut-off value of the NLR

\begin{tabular}{|c|c|c|c|c|}
\hline Characteristic & N (\%) & $\begin{array}{l}\text { NLR }>2.45 \\
(n=40)\end{array}$ & $\begin{array}{l}\mathrm{NLR} \leq 2.45 \\
(n=42)\end{array}$ & $P$ \\
\hline Age in years & & & & 0.004 \\
\hline$>45$ & $21(25.6 \%)$ & $16(40.0 \%)$ & $5(11.9 \%)$ & \\
\hline$\leq 45$ & 61 (74.4\%) & $24(60.0 \%)$ & 37 (88.1\%) & \\
\hline Sex & & & & 0.345 \\
\hline Male & 33 (40.2\%) & $14(35.0 \%)$ & 19 (45.2\%) & \\
\hline Female & 49 (59.8\%) & $26(65.0 \%)$ & $23(54.8 \%)$ & \\
\hline Tumour size & $5.94 \pm 2.65$ & $7.0 \pm 3.0$ & $4.0 \pm 1.1$ & $<0.001$ \\
\hline Location & & & & 0.377 \\
\hline Left & 31 (37.8\%) & $18(45.0 \%)$ & $23(54.8 \%)$ & \\
\hline Right & $51(62.2 \%)$ & $22(55.0 \%)$ & $19(45.2 \%)$ & \\
\hline Symptoms & & & & 0.477 \\
\hline Asymptomatic & $48(58.5 \%)$ & $25(62.5 \%)$ & $23(54.8 \%)$ & \\
\hline Symptomatic & $34(41.5)$ & $15(37.5 \%)$ & $19(45.2 \%)$ & \\
\hline Fuhrman grade & & & & 0.011 \\
\hline 1 to 2 & 32 (39.0\%) & $10(25 \%)$ & $22(52.4 \%)$ & \\
\hline 3 to 4 & $50(61.0 \%)$ & $30(75 \%)$ & $20(47.6 \%)$ & \\
\hline TNM stage & & & & $<0.001$ \\
\hline$|-| \mid$ & 49 (59.8\%) & $14(35.0 \%)$ & $35(83.3 \%)$ & \\
\hline III-IV & $33(40.2 \%)$ & $26(65.0 \%)$ & 7 (16.7\%) & \\
\hline pT status & & & & 0.001 \\
\hline $\mathrm{T} 1-\mathrm{T} 2$ & 64 (78.0\%) & $25(62.5 \%)$ & 39 (92.9\%) & \\
\hline T3-T4 & 18 (22.0\%) & $15(37.5 \%)$ & $3(7.1 \%)$ & \\
\hline pN status & & & & $<0.001$ \\
\hline NO & 59 (72.0\%) & $21(52.5 \%)$ & 38 (90.5\%) & \\
\hline N1 & $23(28.0 \%)$ & $19(47.5 \%)$ & $4(9.5 \%)$ & \\
\hline pM status & & & & 0.112 \\
\hline MO & 78 (95.1\%) & $36(90.0 \%)$ & $42(100.0 \%)$ & \\
\hline M1 & $4(4.9 \%)$ & $4(10.0 \%)$ & $0(0.0 \%)$ & \\
\hline Surgical treatment & & & & 0.307 \\
\hline Radical & $55(67.1 \%)$ & $29(72.5 \%)$ & $26(61.9 \%)$ & \\
\hline Partial & 27 (32.9) & $11(27.5)$ & $16(38.1 \%)$ & \\
\hline Tumour thrombus & & & & 0.014 \\
\hline Negative & $72(87.8 \%)$ & $31(77.5 \%)$ & $41(97.6 \%)$ & \\
\hline Positive & $10(12.2 \%)$ & $9(22.5 \%)$ & $1(2.4 \%)$ & \\
\hline CRP/Alb & & & & $<0.001$ \\
\hline$\leq 0.083$ & $44(53.7 \%)$ & $13(32.5 \%)$ & $31(73.8 \%)$ & \\
\hline$>0.083$ & $38(46.3 \%)$ & $27(67.5 \%)$ & $11(26.2 \%)$ & \\
\hline PLR & & & & 0.001 \\
\hline$\leq 140$ & $46(56.1 \%)$ & $15(37.5 \%)$ & $31(73.8 \%)$ & \\
\hline$>140$ & $36(43.9 \%)$ & $25(62.5 \%)$ & $11(26.2 \%)$ & \\
\hline $\mathrm{LDH}$ & & & & 0.014 \\
\hline Normal & 68 (82.9\%) & 29 (72.5\%) & 39 (92.9\%) & \\
\hline
\end{tabular}

Table 2 Clinicopathological features of Xp11.2 tRCC patients stratified by the cut-off value of the NLR (Continued)

\begin{tabular}{lllll}
\hline Characteristic & $\mathrm{N}(\%)$ & $\begin{array}{l}\mathrm{NLR}>2.45 \\
(n=40)\end{array}$ & $\begin{array}{l}\mathrm{NLR} \leq 2.45 \\
(n=42)\end{array}$ & $P$ \\
\hline Elevated & $14(17.1 \%)$ & $11(27.5 \%)$ & $3(7.1 \%)$ & \\
Proteinuria & & & & 0.013 \\
No & $63(76.8 \%)$ & $26(65.0 \%)$ & $37(88.1 \%)$ & \\
Yes & $19(23.2 \%)$ & $14(35.0 \%)$ & $5(11.9 \%)$ & \\
\hline
\end{tabular}

were calculated accordingly. Survival analyses of OS and DFS were performed using the Kaplan-Meier method, and the log-rank test was performed for the significance comparison. A Cox proportional-hazard model was applied for univariate and multivariate analyses, and hazard ratios (HR) with $95 \%$ confidence intervals $(95 \% \mathrm{CI})$ and $P$ values are presented. If variables were significantly associated with other variables, they were excluded from the final multivariable analysis. A $P$ value $<0.05$ was considered statistically significant in all statistical analyses.

\section{Results}

Patient and tumour characteristics

The clinicopathological characteristics of 82 Xp11.2 tRCC patients are shown in Table 1. Among them, 78 (95.1\%) were diagnosed with a localized mass (T1-3 N0/+ M0), while 4 (4.9\%) had distant metastasis before surgery. After a median follow-up time of 31 months (range: 2 to 108 months), 14 (17\%) died and 34 (41\%) developed recurrence or distant metastasis. Their mean age at surgery was 37 years (range: 2 to 71 months). The 5-year overall survival (OS) was $82.9 \%$ (68/82), 5-year-cancer-specific survival (CSS) was $86.6 \%$ (71/82), and 5-year disease-free survival (DFS) was 61.5\% (48/78).

\section{Optimal cut-off values of inflammatory parameters based on ROC analysis}

Based on ROC analysis, the area under ROC curve (AUC) values of the NLR, CRP/Alb ratio, and PLR were 0.797 ( $P$ $=0.001), 0.772(P=0.003)$, and $0.755(P=0.006)$, respectively, for DFS and the optimal cut-off values for the NLR, CRP/Alb, and PLR were $2.45,0.83$, and 140 , respectively. The corresponding sensitivity and specificity values for DFS were, respectively, 82.4 and $72.8 \%$ for NLR, 70.6 and $72.8 \%$ for CRP/Alb, and 80.0 and $65.9 \%$ for PLR.

\section{Association of the preoperative NLR and clinicopathological characteristics}

Stratified by the cut-off value, the association between the preoperative NLR and clinicopathological characteristics is summarized in Table 2. An elevated NLR was significantly associated with the tumour size $(P<0.001)$, Fuhrman-grade $(P=0.011)$, TNM stage $(P<0.001)$, pT status $(P=0.001)$, pN status $(P<0.001)$, tumour thrombus 
Table 3 Clinical-pathological features of Xp11.2 tRCC patients stratified by the cut-off values of the CRP/Alb ratio and PLR

\begin{tabular}{|c|c|c|c|c|c|c|c|}
\hline Characteristic & N (\%) & $\begin{array}{l}\text { CRP/Alb> } 0.083 \\
(n=39)\end{array}$ & $\begin{array}{l}\text { CRP/Alb } \leq 0.083 \\
(n=43)\end{array}$ & $P$ & $\begin{array}{l}\text { PLR }>140 \\
(n=36)\end{array}$ & $\begin{array}{l}\text { PLR } \leq 140 \\
(n=46)\end{array}$ & $P$ \\
\hline Age in years & & & & 0.011 & & & 0.015 \\
\hline$>45$ & $21(25.6 \%)$ & 15 (38.5\%) & $6(14.0 \%)$ & & 14 (38.9\%) & 7 (15.2\%) & \\
\hline$\leq 45$ & $61(74.4 \%)$ & $24(61.5 \%)$ & 37 (86.0\%) & & $22(61.1 \%)$ & 39 (84.8\%) & \\
\hline Sex & & & & 0.224 & & & 0.113 \\
\hline Male & $33(40.2 \%)$ & 13(33.3\%) & $20(46.5 \%)$ & & $11(30.6 \%)$ & $22(47.8 \%)$ & \\
\hline Female & $49(59.8 \%)$ & $26(66.7 \%)$ & $23(53.5 \%)$ & & $25(69.4 \%)$ & $24(52.2 \%)$ & \\
\hline Tumor size & $5.94 \pm 2.65$ & $6.7 \pm 3.0$ & $4.8 \pm 2.1$ & 0.035 & $7.4 \pm 3.1$ & $4.1 \pm 1.2$ & $<0.001$ \\
\hline Location & & & & 0.052 & & & 0.858 \\
\hline Left & $31(37.8 \%)$ & $19(48.7 \%)$ & 12 (27.9\%) & & 14 (38.9\%) & 17 (37.0\%) & \\
\hline Right & $51(62.2 \%)$ & $20(51.3 \%)$ & $31(72.1 \%)$ & & $22(61.1 \%)$ & $29(63.0 \%)$ & \\
\hline Symptoms & & & & 0.412 & & & 0.165 \\
\hline Asymptomatic & $48(58.5 \%)$ & $21(53.8 \%)$ & $27(62.8 \%)$ & & 18 (50.0\%) & 30 (65.2\%) & \\
\hline Symptomatic & $34(41.5 \%)$ & 18 (46.2\%) & 16 (37.2\%) & & 18 (50.0\%) & $16(34.8 \%)$ & \\
\hline Fuhrman grade & & & & 0.018 & & & 0.006 \\
\hline 1 to 2 & $32(39.0 \%)$ & 10 (25.6\%) & $22(51.2 \%)$ & & $8(22.2 \%)$ & $24(52.2 \%)$ & \\
\hline 3 to 4 & $50(61.0 \%)$ & $29(74.4 \%)$ & $21(48.8 \%)$ & & $28(77.8 \%)$ & $22(47.8 \%)$ & \\
\hline TNM stage & & & & 0.004 & & & $<0.001$ \\
\hline$|-| \mid$ & 49 (59.8\%) & $17(43.6 \%)$ & $32(74.4 \%)$ & & $12(33.3 \%)$ & 37 (80.4\%) & \\
\hline III-IV & $33(40.2 \%)$ & $22(56.4 \%)$ & $11(25.6 \%)$ & & $24(66.7 \%)$ & 9 (19.6\%) & \\
\hline pT status & & & & 0.018 & & & 0.006 \\
\hline $\mathrm{T} 1-\mathrm{T} 2$ & 64 (78.0\%) & $26(66.7 \%)$ & 38 (88.4\%) & & $22(61.1 \%)$ & 42 (91.3\%) & \\
\hline T3-T4 & 18 (22.0\%) & $13(33.3 \%)$ & $5(11.6 \%)$ & & 14 (38.9\%) & $4(8.7 \%)$ & \\
\hline pN status & & & & 0.003 & & & 0.003 \\
\hline NO & $59(72.0 \%)$ & $22(56.4 \%)$ & $37(86.0 \%)$ & & $20(55.6 \%)$ & $39(84.8 \%)$ & \\
\hline N1 & $23(28.0 \%)$ & 17 (43.6\%) & $6(14.0 \%)$ & & $16(44.4 \%)$ & $7(15.2 \%)$ & \\
\hline pM status & & & & 0.101 & & & 0.199 \\
\hline MO & $78(95.1 \%)$ & 35 (89.7\%) & $43(100.0 \%)$ & & $33(91.7 \%)$ & $45(97.8 \%)$ & \\
\hline M1 & $4(4.9 \%)$ & $4(10.3 \%)$ & $0(0.0 \%)$ & & $3(8.3 \%)$ & $1(2.2 \%)$ & \\
\hline Surgical treatment & & & & 0.502 & & & 0.686 \\
\hline Radical & $55(67.1 \%)$ & $23(59.0 \%)$ & $22(51.2 \%)$ & & $25(69.4 \%)$ & $30(65.2 \%)$ & \\
\hline Partial & 27 (32.9) & $16(41.0 \%)$ & $11(48.8 \%)$ & & $11(30.6 \%)$ & $16(34.8 \%)$ & \\
\hline Tumour thrombus & & & & 0.011 & & & 0.001 \\
\hline Negative & $72(87.8 \%)$ & $30(76.9 \%)$ & $42(97.7 \%)$ & & $26(72.2 \%)$ & $46(100.0 \%)$ & \\
\hline Positive & $10(12.2 \%)$ & 9 (23.1\%) & $1(2.3 \%)$ & & $10(27.8 \%)$ & $0(0.0 \%)$ & \\
\hline CRP/Alb & & & & - & & & $<0.001$ \\
\hline$\leq 0.083$ & $44(53.7 \%)$ & - & - & & $11(30.6 \%)$ & $33(71.7 \%)$ & \\
\hline$>0.083$ & $38(46.3 \%)$ & - & - & & $25(69.4 \%)$ & $13(28.3 \%)$ & \\
\hline PLR & & & & 0.009 & & & - \\
\hline$\leq 140$ & $46(56.1 \%)$ & $16(41.0 \%)$ & $30(69.8 \%)$ & & - & - & \\
\hline$>140$ & $36(43.9 \%)$ & $23(59.0 \%)$ & $13(30.2 \%)$ & & - & - & \\
\hline $\mathrm{LDH}$ & & & & 0.011 & & & 0.023 \\
\hline Normal & 68(82.9\%) & $28(71.8 \%)$ & 40 (93.0\%) & & $26(72.2 \%)$ & $42(91.3 \%)$ & \\
\hline
\end{tabular}


Table 3 Clinical-pathological features of Xp11.2 tRCC patients stratified by the cut-off values of the CRP/Alb ratio and PLR (Continued)

\begin{tabular}{lllllll}
\hline Characteristic & $\mathrm{N}(\%)$ & $\begin{array}{l}\text { CRP/Alb>0.083 } \\
(n=39)\end{array}$ & $\begin{array}{l}\text { CRP/Alb } \leq 0.083 \\
(n=43)\end{array}$ & $P$ & $\begin{array}{l}\text { PLR }>140 \\
(n=36)\end{array}$ & $\begin{array}{l}\text { PLR } \leq 140 \\
(n=46)\end{array}$ \\
\hline $\begin{array}{l}\text { Elevated } \\
\text { Proteinuria }\end{array}$ & $14(17.1 \%)$ & $11(28.2 \%)$ & $3(7.0 \%)$ & $<0.001$ & $10(27.8 \%)$ & $4(8.7 \%)$ \\
No & & & $20(55.6 \%)$ & $43(93.5 \%)$ \\
Yes & $63(76.8 \%)$ & $22(56.4 \%)$ & $41(95.3 \%)$ & $16(44.4 \%)$ & $3(6.5 \%)$ \\
\hline
\end{tabular}

$(P=0.014)$, CRP/Alb $(P<0.001)$, PLR $(P=0.001)$, LDH $(P$ $=0.014)$ and proteinuria $(P<0.013)$. For patients in the high NLR group, only $35.0 \%$ of patients were at stage I/II and $65.0 \%$ of patients were at stage III-IV $(P<0.001)$, $62.5 \%$ of patients were at stage $\mathrm{T} 1 / \mathrm{T} 2$, and $37.5 \%$ of patients were at stage T3/T4 $(P=0.001)$. However, for patients in the low NLR group, $83.3 \%$ of patients were at stage I/II, $16.7 \%$ were at stage III-IV $(P<0.001), 92.9 \%$ of patients were at stage $\mathrm{T} 1 / \mathrm{T} 2$, and $7.1 \%$ of patients were at stage T3/T4 $(P=0.001)$. Meanwhile, the percentage values of patients at stage N0/N1 were $52.5 \%$ / 47.2\%, and the percentage values of patients negative/positive tumour thrombus were $77.5 \% / 22.5 \%$ in the high NLR group. By comparison, the percentage values of patients at stage N0/ N1 were $90.5 \% / 9.5 \%$, and the percentage values of patients with negative/positive tumour thrombus were 97.6\%/2.4\% in the low NLR group. These results revealed that a high NLR was associated with tumour progression and a low NLR was associated with early-stage of Xp11.2 tRCC. Similarly, Clinicopathological features of Xp11.2 RCC patients stratified by the cut-off value of the CRP/Alb ratio, PLR are summarized in the Table 3.

Univariate and multivariate analyses for both DFS and OS The results of univariate and multivariate analyses for both DFS and OS are shown in Tables 4 and 5. Univariate analysis demonstrated that age, the Fuhrman grade, pT status, pN status, tumour thrombus, the NLR, the CRP/Alb and the PLR were significant predictors for both DFS and OS in Xp11.2 tRCC patients. Multivariable analysis showed that the NLR (HR: 4.25, 95\%, CI 1.19-15.18, $P=0.026$ ) was an independent predictor of DFS in patients with Xp11.2 tRCC, along with pT status $(P<0.001)$, pT status $(P=0.019)$ and age $(P=0.004)$, and the NLR (HR: 26.26; 95\% CI: $1.44-480.3 ; P=0.028$ ) also was an independent predictor of OS in patients with Xp11.2 tRCC, along with age $(P=0.016)$ and a tumour thrombus $(P=0.007)$.

\section{The relationships of the preoperative NLR, pT status, pN status, age and tumour thrombus with survival}

The relationships of the independent predictors in multivariate analyses for DFS and OS, such as the preoperative NLR, pT status, pN status, age and tumour thrombus with survival (OS: $n=82$; DFS: $n=78$ ) were investigated, and the results are shown in Fig. 2. Patients with a preoperative higher NLR had a significantly worse rate of survival than those with a lower NLR ratio with regarding both OS and DFS (Mean OS 49.0 months vs 99.7 months, respectively, log-rank $P=0.009$; Mean DFS 24.5 months vs 90.2 months, respectively, log-rank $P<0.001)$. Patients age $>45$ years had a significantly worse rate of survival than those with age $\leq 45$ years with regarding both OS

Table 4 Univariate and multivariate analyses for variables considered for disease-free survival (DFS) (Cox proportional hazard regression model) $(n=78)$

\begin{tabular}{|c|c|c|c|c|}
\hline \multirow[t]{2}{*}{ Variables } & \multicolumn{2}{|l|}{ Univariate analysis } & \multicolumn{2}{|l|}{ Multivariate analysis } \\
\hline & $\mathrm{HR}(95 \% \mathrm{Cl})$ & $P$ & $\mathrm{HR}(95 \% \mathrm{Cl})$ & $P$ \\
\hline Age $(>45)$ & 2.34 (1.03 to 5.30$)$ & 0.043 & 6.25 (1.78 to 21.97$)$ & 0.004 \\
\hline Symptoms (yes) & 1.06 (0.51 to 2.19$)$ & 0.885 & & \\
\hline Gender (male) & $0.76(0.36$ to 1.64$)$ & 0.490 & & \\
\hline Fuhrman Grade (G3-G4) & 5.24 (2.12 to 12.96$)$ & $<0.001$ & 1.83 (0.64 to 5.24$)$ & 0.261 \\
\hline pT status (T3-T4) & 6.48 (2.96 to 14.19$)$ & $<0.001$ & 6.84 (2.35 to 19.90$)$ & $<0.001$ \\
\hline pN status (N1) & 5.21 (2.52 to 10.77$)$ & $<0.001$ & $3.40(1.22$ to 9.43$)$ & 0.019 \\
\hline Tumour thrombus (yes) & 12.47 (4.81 to 32.34$)$ & $<0.001$ & 2.90 (0.73 to 11.48$)$ & 0.129 \\
\hline $\operatorname{NLR}(>2.45)$ & 4.98 (2.12 to 11.66$)$ & $<0.001$ & 4.25 (1.19 to 15.18$)$ & 0.026 \\
\hline CRP/Alb (>0.083) & $2.90(1.37$ to 6.13$)$ & 0.005 & 1.40 (0.43 to 4.54$)$ & 0.574 \\
\hline $\operatorname{PLR}(>140)$ & $3.76(1.74$ to 8.13$)$ & 0.001 & 1.36 (0.47 to 3.72$)$ & 0.598 \\
\hline
\end{tabular}

Italicized $P$ values are statistically significant 
Table 5 Univariate and multivariate analyses for variables considered for overall survival (OS) (Cox proportional hazard regression model) $(n=82)$

\begin{tabular}{|c|c|c|c|c|}
\hline \multirow[t]{2}{*}{ Variables } & \multicolumn{2}{|l|}{ Univariate analysis } & \multicolumn{2}{|l|}{ Multivariate analysis } \\
\hline & $\mathrm{HR}(95 \% \mathrm{Cl})$ & $P$ & $\mathrm{HR}(95 \% \mathrm{Cl})$ & $P$ \\
\hline Age (> 45 years) & $4.90(1.44$ to 16.69$)$ & 0.011 & 26.56 (1.85 to 380.7) & 0.016 \\
\hline Symptoms (yes) & 2.04 (0.70 to 5.93$)$ & 0.189 & & \\
\hline Gender (male) & 2.14 (0.48 to 9.63$)$ & 0.320 & & \\
\hline Fuhrman Grade (G3-G4) & $1.92(1.04$ to 3.55$)$ & 0.037 & 0.30 (0.07 to 1.28$)$ & 0.103 \\
\hline pT status (T3-T4) & 2.21 (1.24 to 3.95$)$ & 0.007 & 1.82 (0.70 to 4.70$)$ & 0.217 \\
\hline pN status (N1) & 6.22 (2.06 to 18.82 ) & 0.001 & 2.03 (0.20 to 20.45$)$ & 0.547 \\
\hline Tumour thrombus (yes) & 22.32 (6.76 to 73.72 ) & $<0.001$ & 47.40 (2.92 to 769.9$)$ & 0.007 \\
\hline $\operatorname{NLR}(>2.45)$ & 4.46 (1.33 to 14.97$)$ & 0.016 & 26.26 (1.44 to 480.3$)$ & 0.028 \\
\hline CRP/Alb (>0.083) & $23.51(2.90$ to 190.51$)$ & 0.003 & 6.65 (0.35 to 127.0$)$ & 0.208 \\
\hline $\operatorname{PLR}(>140)$ & 4.30 (1.34 to 13.82$)$ & 0.014 & 0.14 (0.01 to 1.67$)$ & 0.120 \\
\hline
\end{tabular}

Italicized $P$ values are statistically significant

and DFS (mean DFS 12.1 months vs 75.0 months, respectively, log-rank $P=0.005$; mean OS 30.5 months vs 67.8 months, respectively, $\log$-rank $P=0.035$ ). Patients at T3-T4 stage and N1 stage had a significantly worse rate of survival than those with at stage T1-T2 and N0 stage regarding DFS (mean DFS 24.5 months vs 90.2 months, respectively, $\log$-rank $P<0.001$; mean DFS 20.4 months vs 81.9 months, respectively, log-rank $P<0.001$ ). Patients positive for a tumour thrombus had a significantly worse rate of survival than those who were negative for a tumour thrombus regarding OS (mean DFS 24.5 months vs 90.2 months, respectively, $\log -\operatorname{rank} P<0.001$ ).

\section{Discussion}

In this multicentre retrospective study, we investigated the prognostic values of the NLR, CRP/Alb ratio, and PLR in 82 Xp11.2 tRCC patients who underwent radical or partial nephrectomy. The results demonstrated that the NLR, CRP/Alb ratio and PLR were all significant predictors and that the NLR was an independent prognostic marker for patients with Xp11.2 tRCC.

Increasing evidence has revealed the involvement of systemic inflammation in cancer development and progression. Neutrophils were shown to produce pro-angiogenic factors such as vascular endothelial growth factor to stimulate tumour development and progression [19]. Moreover, the cytokines involved in cancer-related inflammation, IL-6 and TNF $\alpha$, may induce neutrophilia [20, 21]. Additionally, relative lymphocytopenia may reflect a lower count of CD4+ T-helper lymphocytes, resulting in a suboptimal lymphocyte-mediated immune response to malignancy [22]. Therefore, an NLR may reflect the combined prognostic information of these two inflammatory factors, and a high NLR has been validated as a poor prognostic factor for several different human cancers [10], including clear cell RCC and non-clear cell RCC [14, 17, 22-24].
C-reactive protein (CRP) is a prototype acute phase protein that was demonstrated to be produced in hepatocytes and regulated by growth factors in the malignant tumors such as IL-6 [25]. An elevated CRP level was reported to be associated with a poorer prognosis in various types of human cancers [25-27]. A study conducted by Guo et al. [16] summarized the potential mechanisms regarding how CRP is associated with cancer in follow several aspects:(1). An increased CRP level is created by the tissue inflammation, which is caused by the tumour growth; (2). Tumour antigens activate the immune responses, leading to increased CRP level; (3). Tumour cells increase CRP expression by producing inflammatory proteins, including CRP or by enhancing the role of inflammatory cytokines such as IL-6 and IL-8, which could indirectly increase the CRP level. More recently, several publications demonstrated that the CRP/Alb ratio could be used to predict the prognosis of several cancers [11, 28, 29], and two additional studies confirmed the prognostic value of the CRP/Alb ratio in RCC patients $[16,30]$.

Since the possible association between an increased platelet level and cancer metastasis was first described in 1968 [31], an increased PLT level was confirmed to be a prognostic marker for several cancers, including RCC [32, 33]. Furthermore, Emerging evidence has shown that the platelet-to-lymphocyte ratio (PLR) can be used to assess the response to systemic inflammation and RCC prognosis $[12,15,16]$.

In this study, we explored the relationships of the NLR, CRP/Alb ratio and PLR with survival in Xp11.2 tRCC patients. Compared with the other systemic inflammatory markers, the NLR, CRP/Alb ratio and PLR had better predictive value for DFS (Fig. 1, Tables 2 and 3). Among of them, the NLR had the highest AUC value $(P=0.001)$. The optimal cut-off value of the NLR was 2.45 , which is little lower than the cut-off values of two other studies, 
a

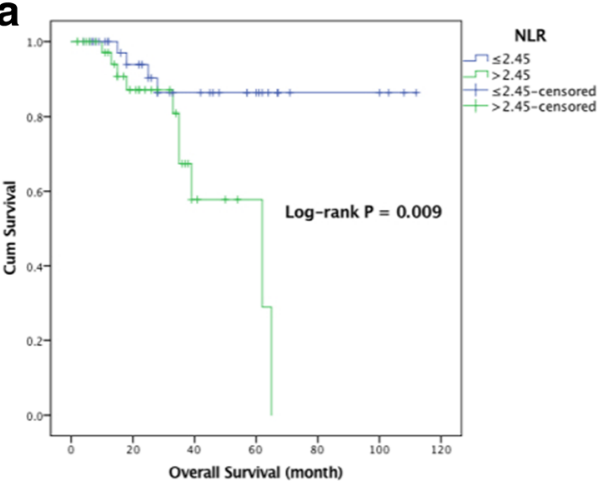

C

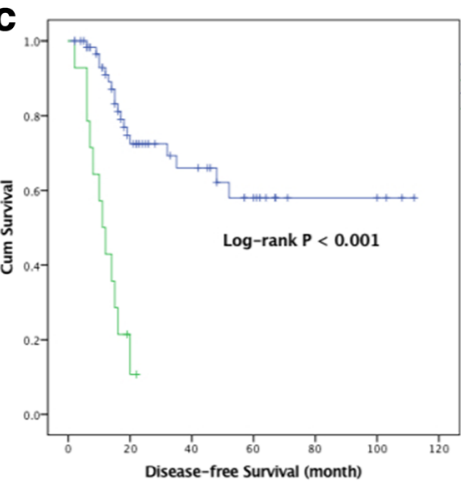

e

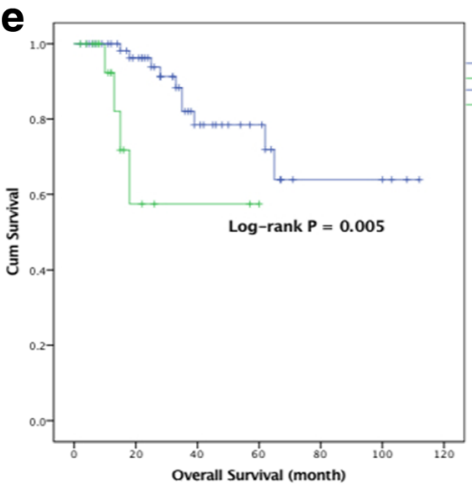

g

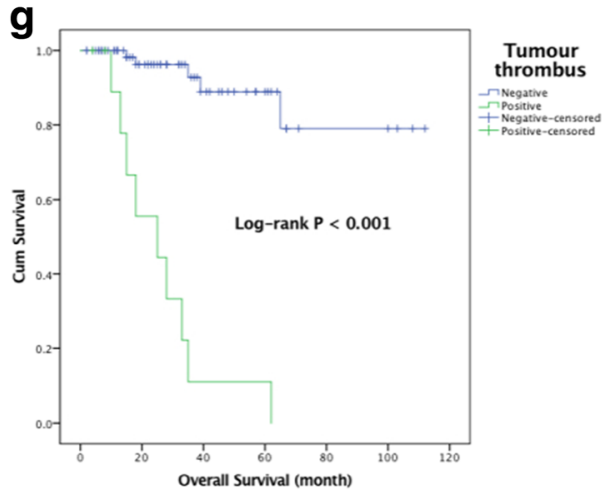

b

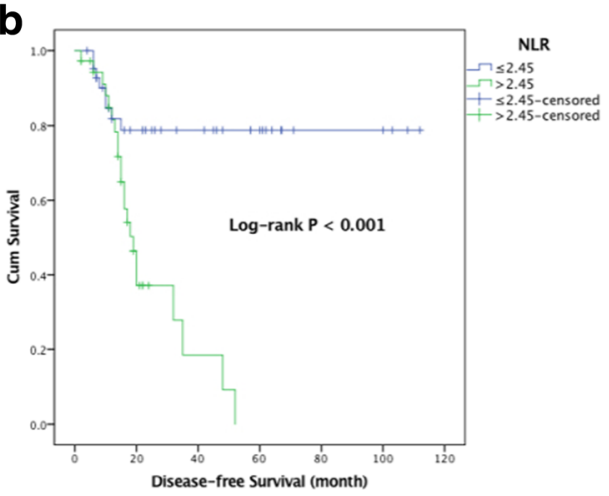

d

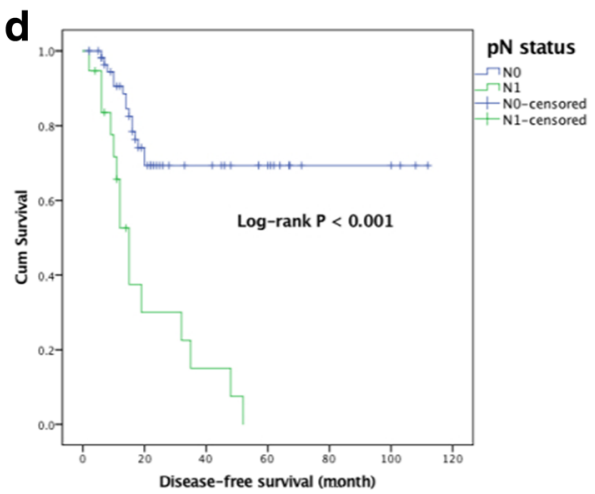

f

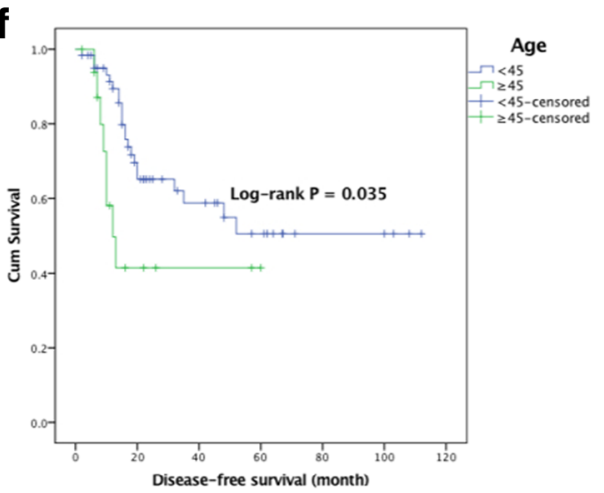

Fig. 2 Kaplan-Meier curves for independent predictors in multivariate analysis regarding DFS ( $n=78)$ and OS ( $n=82)$. a: OS stratified by the NLR; b: DFS stratified by the NLR; c: DFS stratified by PT status; $\mathbf{d}$ : DFS stratified by pN status; $\mathbf{e}$ : OS stratified by age; $\mathbf{f}$ : DFS stratified by age; $\mathbf{g}$ : OS stratified by tumour thrombus 
whose cut-off values were 2.7 and 3.3, respectively $[14,24]$. We consider these differences to be due to the small size of our patients and uniqueness of this tumour. Regarding the CRP/Alb ratio and PLR, the optimal cut-off values were 0.083 and 140, respectively, similar to those reported in previous studies on RCC [15, 16]. Univariate analyses for both DFS and OS showed that a higher NLR, $\mathrm{CRP} / \mathrm{Alb}$ ratio and PLR were all associated with a poorer prognosis of Xp11.2 tRCC patients (Tables 4 and 5), and multivariate analyses showed that only the NLR independently predicted the DFS of patients with Xp11.2 tRCC (HR: 4.25; 95\% CI: $1.19-15.18 ; P=0.026)$ along with the pT status $(P<0.001)$, pN status $(P=0.019)$ and age $(0.014)$ (Table 4), and the NLR (HR: 26.26; 95\% CI: 1.44480.3; $P=0.028$ ) as well as independently predicted the OS of patients with Xp11.2 tRCC, along with age $(P$ $=0.016)$ and tumour thrombus $(P=0.007)$ (Table 5). Our previous studies on Xp11.2 tRCC confirmed that advanced TNM stage and tumour thrombus are the most significant factors that predict a poor prognosis in Xp11 tRCC [4, 34], and we believe that pT status and N status contribute to the advanced TNM stage in the present study. In addition, Kaplan-Meier survival analysis suggested that the patients with a preoperative higher NLR, T3-T4 stage, N1 stage and age $>45$ years had a significantly shorter DFS than those with a lower NLR, T1-T2 stage, N0 stage and age $\leq 45$ years, individually. The patients with a higher NLR, age $>45$ years and positive for tumour thrombus had a significantly shorter OS than those with a lower NLR, age $\leq 45$ years and negative for tumour thrombus. Therefore, a low NLR is associated with the early-stage Xp11.2 tRCC and a high NLR indicates advanced-stage Xp11.2 tRCC, suggesting that the NLR could be a new prognostic indicator related to the progression of Xp11.2 tRCC.

Our findings demonstrate that the NLR, CRP/Alb ratio and PLR were all associated with a poor prognosis in Xp11.2 tRCC patients. Among them, only the NLR independently predicted surgical outcomes of Xp11.2 tRCC patients. These results are important for clinicians to make clinical decisions. According to these preoperative inflammatory markers, patients at high risk can be selected for further treatment and management. With these prognostic factors, more suitable preoperative therapies and more frequent follow-up strategies can be considered for certain high-risk patients with Xp11.2 tRCC.

In addition, the prognostic value of inflammatory markers has never been reported with Xp11.2 tRCC patients. We may, for the first time, predict the surgical outcomes of Xp11.2 tRCC patients using the NLR, CRP/Alb ratio and PLR. Moreover, the sample size of this study was the largest among studies of this tumour worldwide.
To the best of our knowledge, this is the first study that focused on the prognostic values of the NLR, CRP/Alb ratio and PLR in patients with Xp11.2 tRCC. However, this study possesses several limitations. First, our study is a retrospective study, which may limit the prognostic values of the NLR, CRP/Alb ratio and PLR. Therefore, a large-scale prospective study is needed to validate our results. Second, due to the low incidence of Xp11.2 tRCC, our sample size was relatively small, warranting a large-scale study. Third, several other factors that are influential to inflammation such as smoking habits and life styles were not included in the study.

\section{Conclusions}

In summary, we found that the NLR, CRP/Alb ratio and PLR were all potential markers for the survival of Xp11.2 tRCC; thus, they could be considered for clinical decision-making. Among them, the NLR is an independent predictor of both DFS and OS for patients with $\mathrm{Xp11.2} \mathrm{tRCC} \mathrm{and} \mathrm{can} \mathrm{be} \mathrm{used} \mathrm{to} \mathrm{predict} \mathrm{the} \mathrm{surgical}$ outcomes of Xp11.2 tRCC patients who underwent full resection.

\section{Abbreviations \\ Alb: Albumin; CRP: C-reactive protein; CRP/Alb: C-reactive protein/albumin; DFS: Disease-free survival; HR: Hazard ratio; LC: Lymphocyte count; \\ LDH: Lactate dehydrogenase level; NC: Neutrophil count; NLR: Neutrophil count to lymphocyte count ratio; OS: Overall survival; PLR: Platelet count to lymphocyte count ratio; PLT: Platelet count; RCC: Renal cell carcinoma; ROC: Receive operating characteristic; TFE3: Transcription factor E3; Xp11.2 tRCC: Xp11.2 translocation/TFE3 gene fusions Renal Cell Carcinoma}

\section{Acknowledgements}

We thank Gutian Zhang, Xiaogong Li, Linfeng Xu and Xiaozhi Zhao (Nanjing Drum Tower Hospital) for providing patient information, and Jie Gao, Zhen Wang and Wenliang Ma (Nanjing Drum Tower Hospital) for providing technical assistance.

\section{Funding}

This research was supported by the National Natural Science Foundation of China (ID: 81572512,81772710 and 81572519) and Nanjing Medical Science and Technique Development Foundation (ID: QRX17049). The funders played no roles in the design of the study and collection, analysis, and interpretation of data and in writing the manuscript.

\section{Availability of data and materials}

The datasets of the current study are available from the corresponding author on reasonable request.

\section{Authors' contributions \\ SA: Project development, Data collection, Data analysis, Manuscript writing and editing. FQ: Project development, Data collection, Data analysis, Manuscript editing. NL: Data analysis, Results interpretation, Manuscript editing. JS: Data collection, Data analysis, Results interpretation. WX: Data collection, Data analysis, Results interpretation. LHZ: Data collection, Data analysis, Results interpretation. HQG: Data collection, Results interpretation, Manuscript editing. WDG: Project development, Data analysis, Manuscript writing and editing. All authors read and approved the final manuscript.}

\section{Ethics approval and consent to participate}

The study received the ethics approval from the Ethics Committee of Nanjing Drum Tower Hospital, Jiangsu Province Hospital, Jiangsu Cancer Hospital and Zhongda Hospital Southeast University. 
All procedures performed in the study involving human participants were in accordance with the ethical standards of the institutional research committee and with the 1964 Helsinki declaration and its later amendments or comparable ethical standard.

All patients agreed and signed the informed consent to participate in the study, and they agreed that their information (including clinical information, surgical related data, pathological data, blood laboratory tests and surveillance) would be collected for scientific study and published in professional medical journals.

\section{Competing interests}

The authors declare that they have no competing interests.

\section{Publisher's Note}

Springer Nature remains neutral with regard to jurisdictional claims in published maps and institutional affiliations.

\section{Author details}

'Department of Urology, Nanjing Drum Tower Hospital, The Affiliated Hospital of Nanjing University Medical School, No. 321 Zhongshan Road, Nanjing 210008, Jiangsu Province, China. ²Department of Oncology, Jiangsu Province Hospital, The First Affiliated Hospital of Nanjing Medical University, Nanjing, Jiangsu, China. ${ }^{3}$ Department of Pathology, Jiangsu Cancer Hospital, The Affiliated Cancer Hospital of Nanjing Medical University, Nanjing, Jiangsu, China. ${ }^{4}$ Department of Pathology, Zhongda Hospital Southeast University, Nanjing, Jiangsu, China.

Received: 4 February 2018 Accepted: 31 May 2018

Published online: 11 June 2018

\section{References}

1. Ellis $C L$, Eble JN, Subhawong AP, Martignoni G, Zhon M, Ladanyi M, Epstein I, Netto GJ, Argani P. Clinical heterogeneity of Xp11 translocation renal cell carcinoma: impact of fusion subtype, age, and stage. Mod Pathol. 2014; 27(6):875-86.

2. Qu Y, Gu C, Wang H, Chang K, Yang X, Zhou X, Dai B, Zhu Y, Shi G, Zhang $H$, et al. Diagnosis of adults Xp11.2 translocation renal cell carcinoma by immunohistochemistry and FISH assays: clinicopathological data from ethnic Chinese population. Sci Rep. 2016;6:21677.

3. Sukov WR, Hodge JC, Lohse CM, Leibovich BC, Thompson RH, Pearce KE, Wiktor AE, Cheville JC. TFE3 rearrangements in adult renal cell carcinoma: clinical and pathologic features with outcome in a large series of consecutively treated patients. Am J Surg Pathol. 2012;36(5):663-70.

4. Liu N, Wang Z, Gan W, Xiong L, Miao B, Chen X, Guo H, Li D. Renal cell carcinoma associated with Xp11.2 translocation/TFE3 gene fusions: clinical features, treatments and prognosis. PLoS One. 2016;11(11):e0166897.

5. Choo MS, Jeong CW, Song C, Jeon HG, Seo SI, Hong SK, Byun SS, Chung JS, Hong SH, Hwang EC, et al. Clinicopathologic characteristics and prognosis of Xp11.2 translocation renal cell carcinoma: multicenter, propensity score matching analysis. Clin Genitourin Cancer. 2017;15(5):e819-e825.

6. Argani P, Antonescu CR, Illei PB, Lui MY, Timmons CF, Newbury R, Reuter VE, Garvin AJ, Perez-Atayde AR, Fletcher JA, et al. Primary renal neoplasms with the ASPL-TFE3 gene fusion of alveolar soft part sarcoma: a distinctive tumor entity previously included among renal cell carcinomas of children and adolescents. Am J Pathol. 2001;159(1):179-92.

7. Malouf GG, Camparo P, Molinie V, Dedet G, Oudard S, Schleiermacher G, Theodore C, Dutcher J, Billemont B, Bompas E, et al. Transcription factor E3 and transcription factor EB renal cell carcinomas: clinical features, biological behavior and prognostic factors. J Urol. 2011:185(1):24-9.

8. Mantovani A, Allavena P, Sica A, Balkwill F. Cancer-related inflammation. Nature. 2008;454(7203):436-44.

9. Coussens LM, Werb Z. Inflammation and cancer. Nature. 2002:420(6917):860-7.

10. Roxburgh CS, McMillan DC. Role of systemic inflammatory response in predicting survival in patients with primary operable cancer. Future Oncol. 2010;6(1):149-63.

11. Kinoshita A, Onoda H, Imai N, Iwaku A, Oishi M, Tanaka K, Fushiya N, Koike K, Nishino H, Matsushima M. The C-reactive protein/albumin ratio, a novel inflammation-based prognostic score, predicts outcomes in patients with hepatocellular carcinoma. Ann Surg Oncol. 2015;22(3):803-10.

12. Smith RA, Bosonnet L, Raraty M, Sutton R, Neoptolemos JP, Campbell F Ghaneh P. Preoperative platelet-lymphocyte ratio is an independent significant prognostic marker in resected pancreatic ductal adenocarcinoma. Am J Surg. 2009;197(4):466-72.

13. Walsh SR, Cook EJ, Goulder F, Justin TA, Keeling NJ. Neutrophil-lymphocyte ratio as a prognostic factor in colorectal cancer. J Surg Oncol. 2005;91(3):181-4

14. Ohno Y, Nakashima J, Ohori M, Hatano T, Tachibana M. Pretreatment neutrophil-to-lymphocyte ratio as an independent predictor of recurrence in patients with nonmetastatic renal cell carcinoma. J Urology. 2010;184(3):873-8.

15. Keskin S, Keskin Z, Taskapu HH, Kalkan H, Kaynar M, Poyraz N, Toy H. Prognostic value of preoperative neutrophil-to-lymphocyte and platelet-tolymphocyte ratios, and multiphasic renal tomography findings in histological subtypes of renal cell carcinoma. BMC Urol. 2014;14:95.

16. Guo SJ, He XB, Chen Q, Yang GW, Yao K, Dong P, Ye YL, Chen D, Zhang ZL, Qin ZK, et al. The C-reactive protein/albumin ratio, a validated prognostic score, predicts outcome of surgical renal cell carcinoma patients. BMC Cancer. 2017;17:171.

17. de Martino M, Pantuck AJ, Hofbauer S, Waldert M, Shariat SF, Belldegrun AS, Klatte T. Prognostic impact of preoperative neutrophil-to-lymphocyte ratio in localized nonclear cell renal cell carcinoma. J Urol. 2013;190(6):1999-2004.

18. Rao Q, Guan B, Zhou XJ. Xp11.2 translocation renal cell carcinomas have a poorer prognosis than non-Xp11.2 translocation carcinomas in children and young adults: a meta-analysis. Int J Surg Pathol. 2010;18(6):458-64.

19. Kusumanto YH, Dam WA, Hospers GA, Meijer C, Mulder NH. Platelets and granulocytes, in particular the neutrophils, form important compartments for circulating vascular endothelial growth factor. Angiogenesis. 2003;6(4): 283-7.

20. Ulich TR, del Castillo J, Guo KZ. In vivo hematologic effects of recombinant interleukin-6 on hematopoiesis and circulating numbers of RBCs and WBCs. Blood. 1989;73(1):108-10

21. Ulich TR, Delcastillo J, Keys M, Granger GA, Ni PX. Kinetics and mechanisms of recombinant human Interleukin-1 and tumor necrosis factor-alphainduced changes in circulating numbers of neutrophils and lymphocytes. J Immunol. 1987;139(10):3406-15.

22. Viers BR, Thompson RH, Boorjian SA, Lohse CM, Leibovich BC, Tollefson MK. Preoperative neutrophil-lymphocyte ratio predicts death among patients with localized clear cell renal carcinoma undergoing nephrectomy. Urol Oncol-Semin Ori. 2014;32(8):1277-84.

23. Pichler M, Hutterer GC, Stoeckigt C, Chromecki TF, Stojakovic T, Golbeck S, Eberhard K, Gerger A, Mannweiler S, Pummer K, et al. Validation of the pretreatment neutrophil lymphocyte ratio as a prognostic factor in a large European cohort of renal cell carcinoma patients. Brit J Cancer. 2013;108(4): 901-7.

24. Ayala AG, Srigley JR, Ro JY, Abdul-Karim FW, Johnson DE. Clear cell cribriform hyperplasia of prostate. Report of 10 cases. Am J Surg Pathol. 1986:10(10):665-71.

25. Nozoe T, Korenaga D, Futatsugi M, Saeki H, Maehara Y, Sugimachi K. Immunohistochemical expression of C-reactive protein in squamous cell carcinoma of the esophagus - significance as a tumor marker. Cancer Lett. 2003;192(1):89-95.

26. Deichmann M, Benner A, Waldmann V, Bock M, Jackel A, Naher $H$. Interleukin-6 and its surrogate C-reactive protein are useful serum markers for monitoring metastasized malignant melanoma. J Exp Clin Cancer Res. 2000;19(3):301-7.

27. Wieland A, Kerbl R, Berghold A, Schwinger W, Mann G, Urban C. C-reactive protein (CRP) as tumor marker in pediatric and adolescent patients with Hodgkin disease. Med Pediatr Oncol. 2003;41(1):21-5.

28. Liu XC, Sun XW, Liu JJ, Kong PF, Chen SX, Zhan YQ, Xu DZ. Preoperative Creactive protein/albumin ratio predicts prognosis of patients after curative resection for gastric Cancer. Transl Oncol. 2015;8(4):339-45.

29. Zhou T, Zhan JH, Hong SD, Hu ZH, Fang WF, Qin T, Ma YX, Yang YP, He XB, Zhao $Y Y$, et al. Ratio of C-reactive protein/albumin is an inflammatory prognostic score for predicting overall survival of patients with small-cell lung Cancer. Sci Rep-Uk. 2015:5:10481.

30. Chen Z, Shao YJ, Fan M, Zhuang QF, Wang K, Cao W, Xu XL, He XZ. Prognostic significance of preoperative $C$-reactive protein: albumin ratio in patients with clear cell renal cell carcinoma. Int J Clin Exp Patho. 2015;8(11): 14893-900.

31. Gasic GJ, Gasic TB, Stewart CC. Antimetastatic effects associated with platelet reduction. P Natl Acad Sci USA. 1968;61(1):46.

32. Long Y, Wang T, Gao Q, Zhou C. Prognostic significance of pretreatment elevated platelet count in patients with colorectal cancer: a meta-analysis. Oncotarget. 2016;7(49):81849-61. 
33. Brookman-May S, May M, Ficarra V, Kainz MC, Kampel-Kettner K, Kohlschreiber S, Wenzl V, Schneider M, Burger M, Wieland WF, et al. Does preoperative platelet count and thrombocytosis play a prognostic role in patients undergoing nephrectomy for renal cell carcinoma? Results of a comprehensive retrospective series. World J Urol. 2013;31(5):1309-16.

34. Xu L, Yang R, Gan W, Chen X, Qiu X, Fu K, Huang J, Zhu G, Guo H. Xp11.2 translocation renal cell carcinomas in young adults. BMC Urol. 2015;15:57.

Ready to submit your research? Choose BMC and benefit from:

- fast, convenient online submission

- thorough peer review by experienced researchers in your field

- rapid publication on acceptance

- support for research data, including large and complex data types

- gold Open Access which fosters wider collaboration and increased citations

- maximum visibility for your research: over $100 \mathrm{M}$ website views per year 